\title{
Effects of Tobacco Pathogens and Their Antagonistic Bacteria on Tobacco Root Exudates
}

\section{Cheng Li ${ }^{*}$, Jun Yu2 ${ }^{2}$, Lu Gan1, Jingguo Sun ${ }^{2}$, Changjun Wang², Qin Wang1, Shouwen Chen"1, Yong Yang1\#}

${ }^{1}$ State Key Laboratory of Biocatalysis and Enzyme Engineering, School of Life Sciences, Hubei University, Wuhan, China

${ }^{2}$ Tobacco Research Institute of Hubei Province, Wuhan, China

Email:"519971800@qq.com

How to cite this paper: Li, C., Yu, J., Gan, L., Sun, J.G., Wang, C.J., Wang, Q., Chen, S.W. and Yang, Y. (2018) Effects of Tobacco Pathogens and Their Antagonistic Bacteria on Tobacco Root Exudates. Open Journal of Applied Sciences, 8, 518-531. https://doi.org/10.4236/ojapps.2018.811042

Received: November 5, 2018

Accepted: November 25, 2018

Published: November 28, 2018

Copyright $\odot 2018$ by authors and Scientific Research Publishing Inc. This work is licensed under the Creative Commons Attribution International License (CC BY 4.0).

http://creativecommons.org/licenses/by/4.0/

\begin{abstract}
The research on relationship between rhizosphere microbes and root exudates has a great significance on discussion of interaction between rhizosphere microbes and plants, as well as control of soil-borne diseases and insect pest. GC-MS was used to analyze changes of tobacco root exudates under the antagonistic action of tobacco bacterial wilt and black shank. It turned out that when pathogens of tobacco bacterial wilt and black shank in tobacco root microorganisms increase, tobacco root exudates augmented rapidly among of which organic acids have the biggest growth, followed by amines. When the pathogens of tobacco bacterial wilt and black shank are inhibited by the active substance of antagonistic antibacterial, 20 - 23 kinds of root exudates are added; besides, the content of 7 substances was reduced to 0 . Another interesting finding was that the fluctuations of phthalic acid, isophthalic acid and benzoic acid, which have caused continuous cropping obstacles, were very distinct. The results of this study have provided novel clues for the exploration of continuous tobacco cropping obstacles and soil-borne diseases.
\end{abstract}

\section{Keywords}

Root Exudates, Rhizosphere Microbes, Allelochemical, GC-MS

\section{Introduction}

Plant-microorganism interaction maintains or dominates the ecological functions of terrestrial ecosystems. Microorganisms in soil environment are regarded

*These authors contributed equally to this work. 
as the largest reservoir of biological diversity in nature [1]. As many as $1011 \mathrm{mi}-$ crobial cells and 30,000 kinds of prokaryotes can be identified from one gram of rhizosphere soil [2] [3]. Rhizosphere microbes [4], considered as the second genome of plants [5], alter the defensive ability of plants by regulating the host immune system [6] [7] or recruit beneficial plant bacteria [8] [9], or inhibit the growth of plant pathogen by changing plant nutrition supply [10]. Therefore, rhizosphere microbes played a vital role in plant growth, health, yield and other aspects of regulation [11]. However, root exudates are the potential and important intermediates in the interaction between rhizosphere microorganisms and plants [12] [13]. The study of the interaction between rhizosphere microorganisms and root exudates is helpful to the study of the interaction between rhizosphere microorganisms and plants. It is of great significance to evaluate plant growth and soil-borne diseases and insect pests.

Recently, the investigation of the relationship between rhizosphere microbes and root exudates mainly focused on the regulation of root exudates on the formation, metabolism, growth and diversity of rhizosphere microorganisms [14] [15]. In fact, rhizosphere microorganisms can affect plant growth by absorbing a specific component of root exudates to stimulate changes in the abundance or quality of root exudates [16] [17] [18] [19]. For example, inoculation with Bacillus subtilis B26 not only increased the yield of rape (B. distachyon), but also increased drought resistance [20], even in the absence of nitrogen source. Both (Azospirillum) and (Herbaspirillum) of Vibrio herbaceus could promote the growth of Brassica napus [21]. These studies suggest that rhizosphere microbes play a potential role in plant root exudates. However, because of the complexity of soil microbes in heterogeneous environments, it is more challenging to explore how soil microbes directly affect root exudates.

In present study, the components of tobacco root exudates under the action of different rhizosphere microorganisms were detected and analyzed by gas chromatography-mass spectrometry (GC-MS), and the changes of tobacco root exudates were discussed. It provides effective information for the study of how microbes change cell membrane permeability and signal transduction, and provides a new clue for the prevention and treatment of tobacco soil-borne diseases.

\section{Materials and Methods}

Tobacco (Yunyan 87) was selected as culture material; Yunyan 87 was selected and bred by Yunnan Tobacco Science Research Institute and China Tobacco Breeding Research (South) Center. It was composed of Yunyan No. 2 as the female parent and K326 as the male parent. Besides, it was approved by the National Variety Approval Committee in December 2000. The main agronomic traits of Yunyan 87 were extremely stable, and its coefficient of variation was smaller than that of the control $\mathrm{k} 326$. The growth was uniform and widely adaptable in the field. The blade has the similar thickness, and the layer is yellow and easy to bake. It has an average yield about $2613 \mathrm{~kg} / \mathrm{hm}^{2}$. The comprehensive evaluation index is superior to the main production variety k326. Yunyan 87 has 
high quality, stable production and wide adaptability, strong resistance to stress, easy to bake and so on. Tobacco bacterial wilt pathogen (Ralstonia solanacearum) and its antagonistic bacteria (Bacillus amyloliquefacien $\mathrm{YH} 22$ ); black shank pathogen (Phytophthora parasitica var. nicotianae) and its antagonistic bacteria (Bacillus amyloliquefacien TU1). All of the above bacteria were preserved in our laboratory.

\subsection{Tobacco Cultivation and Root Exudate Extraction}

First, Tobacco seedling transplanted to peat soil after the Great Cross $(1 \mathrm{~kg}$ peat soil was added with bacterial wilt pathogen and black shank pathogen bacteria solution $50 \mathrm{ml}, 1.0 \times 10^{9} \mathrm{CUF} / \mathrm{mL}$ ), then sampling after 20 days of cultivation. The culture temperature was $30^{\circ} \mathrm{C}$, watering every 5 days and ensure adequate lighting. The remaining samples were supplemented with antagonistic bacteria against tobacco bacterial wilt and black shank (50 ml, $\left.1.0 \times 10^{9} \mathrm{CUF} / \mathrm{mL}\right)$, sampling after 20 days of cultivation too. Collection of tobacco root exudates has adopted the method reported in the literature [22]; repeating 3 times for each experiment and 3 parallels at a time. The collected root exudates were extracted with ethyl acetate: soak each uprooted tobacco with $100 \mathrm{ml}$ distilled water, store in the dark for 48 hours, centrifuge the leachate and then leave the supernatant for suction filtration and adjust $\mathrm{pH}$ of the solution to acidity (pH2), alkalinity (pH11), and neutral ( $\mathrm{pH} 7)$ separately, with the same volume of ethyl acetate, extracting the solution. Then concentrated the extract under reduced pressure to $1 \mathrm{~mL}\left(45^{\circ} \mathrm{C}\right)$, filtered through a $0.45 \mu \mathrm{m}$ filter, and finally collected the organics three times. The solution was transferred to a rotary evaporation flask, and evaporated to dryness at $50^{\circ} \mathrm{C}$. The residuals were stored at low temperature $\left(-80^{\circ} \mathrm{C}\right)$

\subsection{Derivatization of Root Exudates and GC-MS Detection Conditions}

Add mixture of $40 \mu \mathrm{L} 20 \mathrm{mg} / \mathrm{mL}$ methoxyamine salt pyridine and $10 \mu \mathrm{L} 5 \%$ phenylacetate to the freeze-dried root exudates; then incubating in a $60^{\circ} \mathrm{C}$ incubator for $60 \mathrm{~min}$. Finally, $50 \mu \mathrm{L}$ of the silylating reagent N,O-Bis(trimethylsiyl) acetamide (BSTFA) was added and incubated at $70^{\circ} \mathrm{C}$ for $60 \mathrm{~min}$. Add mixture of $40 \mu \mathrm{L} 20 \mathrm{mg} / \mathrm{mL}$ methoxyamine salt pyridine and $10 \mu \mathrm{L} 5 \%$ phenylacetate to the freeze-dried root exudates; then incubating in a $60^{\circ} \mathrm{C}$ incubator for $60 \mathrm{~min}$. Finally, $50 \mu \mathrm{L}$ of the silylating reagent N,O-Bis(trimethylsiyl)acetamide (BSTFA) was added and incubated at $70^{\circ} \mathrm{C}$ for $60 \mathrm{~min}$, cooled to room temperature naturally. GC-MS was analyzed by Thermo Scientific Gas Chromatography/Mass Spectrometer (DSQII). GC condition: the capillary column is TG-5MS (30 $\mathrm{m} \times$ $0.25 \mathrm{~mm} \times 0.25 \mu \mathrm{m}$ ), the inlet temperature is $250^{\circ} \mathrm{C}$, and the split ratio is $50: 1$. The heating program of column temperature: from $50^{\circ} \mathrm{C}$ to $250^{\circ} \mathrm{C}$ at a rate of $3^{\circ} \mathrm{C} / \mathrm{min}$, continue to rise to $250^{\circ} \mathrm{C}$ at a rate of $25^{\circ} \mathrm{C} / \mathrm{min}$, remaining for $4 \mathrm{~min}$. The carrier gas is $\mathrm{He}$ (99.999\%), the flow rate is $20 \mathrm{psi}$, and the injection volume is $1 \mu \mathrm{L}$. MS condition: ionization energy is $70 \mathrm{eV}$, ion source temperature is 
$300^{\circ} \mathrm{C}$, scanning range is $30-600 \mathrm{~m} / \mathrm{z}$ and transmission line temperature is $280^{\circ} \mathrm{C}$.

\subsection{Data Statistics and Analysis}

Processing and analyzing data with Origin 8.0 and SPSS 16.0, and GC-MS data was analyzed by Trace Finder 4.0. Statistics was obtained by Trace Finder Data Library. The relative content of root exudates was calculated by peak area normalization method.

\section{Results and Discussion}

\section{1) Analysis of tobacco root exudates after treating by pathogen of tobacco bacterial wilt and its antagonist.}

The composition of the root exudates of Yunyan 87 was analyzed by GC-MS after irrigating pathogen of bacterial wilt and its antagonist. The main components in the control group were amines, alcohols, acids, lipids, sugars and esters, with the corresponding percentages of $15.78 \%, 36.84 \%, 36.84 \%, 0 \%, 5.26 \%$ and $5.26 \%$. Under the function of pathogen of bacterial wilt, the contents of amines, alcohols, acids, lipids, sugars and esters were 18.42\%, 31.57\%, 44.73\%, 5.26\%, $5.26 \%$ and $5.26 \%$ respectively. While by the antagonistic effect of pathogen of bacterial wilt, the contents of amines, alcohols, acids, lipids, sugars and esters were $11.92 \%, 23.81 \%, 38.09 \%, 9.52 \%, 11.91 \%$ and $4.76 \%$ accordingly (Figure 1 ). There were more than 23 types of root exudates of Yunyan 87 compared with the control group, mainly including 3 kinds of amines, 6 kinds of alcohols, 10 kinds of acids, 2 kinds of sugars, 1 kind of esters and cyclopropene respectively (Table 1). There are the same components of 5 types with contents decrease and 9 types with contents increase. It was noteworthy that the most remarkable increase was terephthalic acid (96.11\%) and benzoic acid (143.75\%). After the addition of antagonistic bacteria of bacterial wilt, there were 28 kinds of components increased among of which 9 were newly appeared and 12 of them decreased among of which 8 were completely disappeared, namely thiodiglycol, 1-butanol, and C. acid, isophthalic acid, myristic acid, ethyl benzoate, azelaic acid and propylene.

According to the analysis, we found that the content of propionic acid, isophthalic acid, isophthalic acid, terephthalic acid, myristic acid, ethyl benzoate, benzoic acid and sebacic acid showed the greatest volatility, the content in the control group was 0 (except for terephthalic acid, the content was 0.7792). The treatment group of pathogen of bacterial wilt reached the highest value, while the content decreased to 0 after the addition of antagonistic bacteria (except for terephthalic acid, from 1.5281 to 0.8589 , with a decrease of $77.91 \%$ ). Totally, amines were significantly decreased by loading $B$. amyloliquefacien $\mathrm{YH} 22$ (T-test, $p=0.045$ ), alcohols content were continuously and significantly decreased by loading $R$. solanacearum and $B$. amyloliquefacien YH22 (T-test, 0.01 $<p<0.05)$, the content of acids and lipids were alwalys significantly increased 


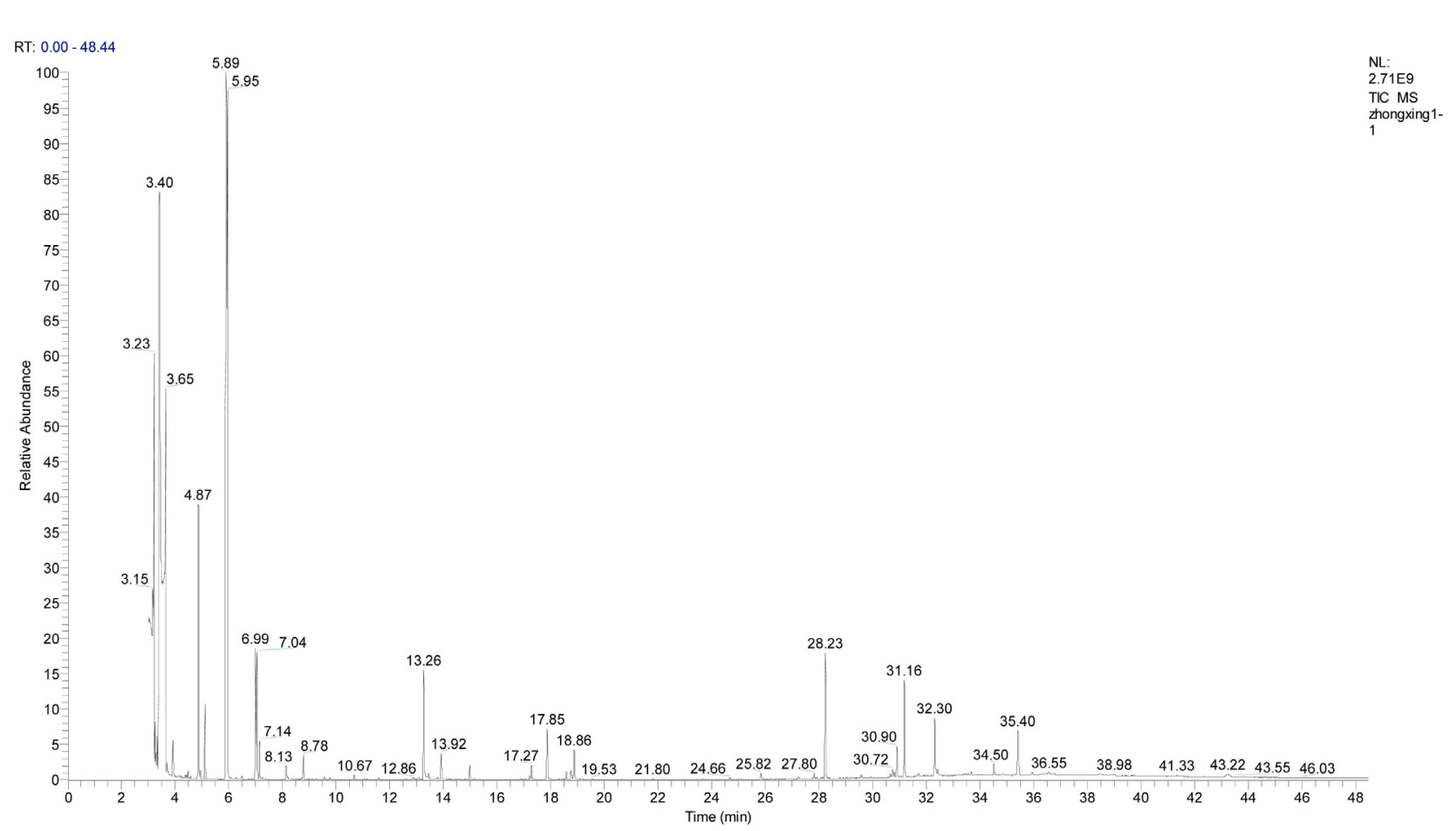

(a)

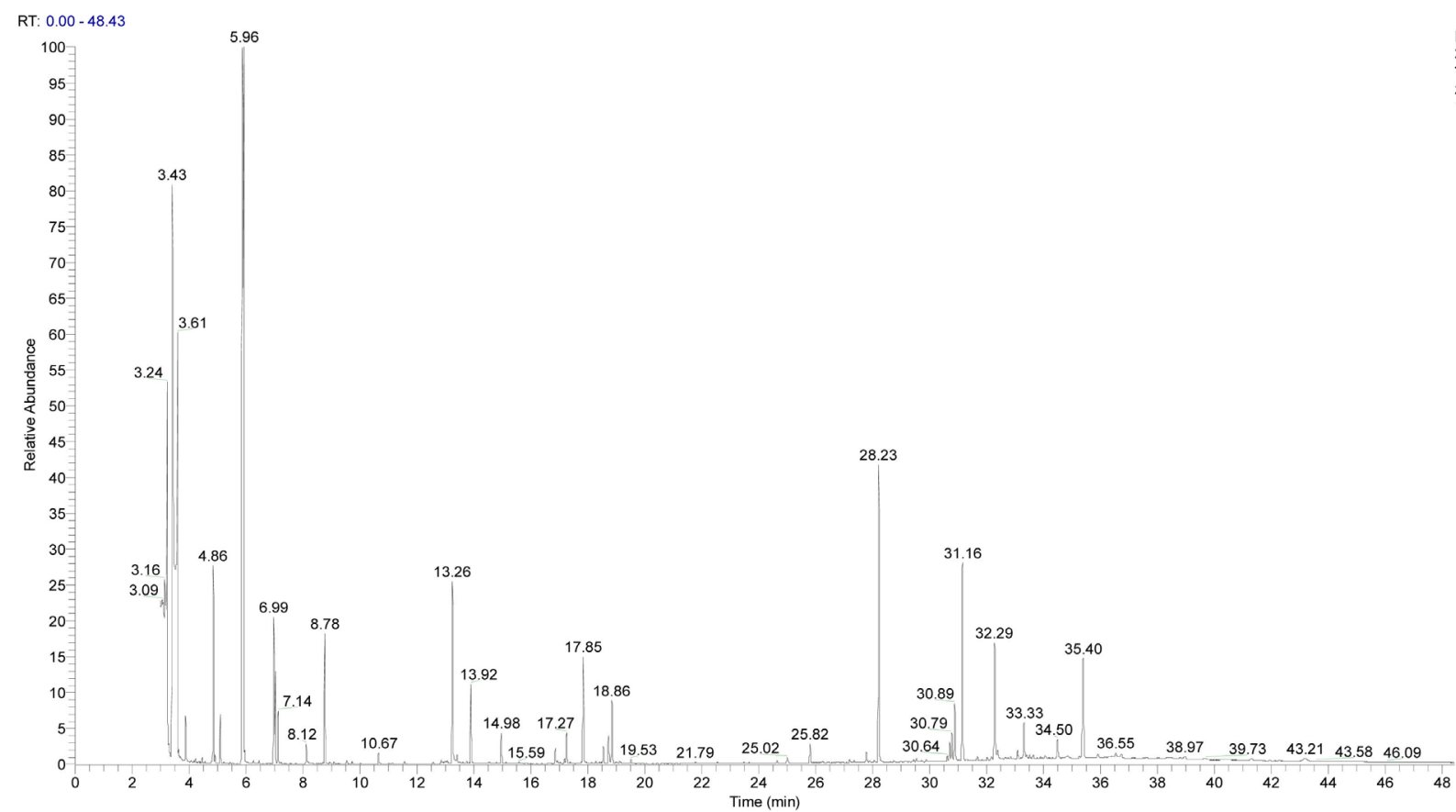

(b)

Figure 1. Total ion current of GC-MS with (a) treatment of pathogen of bacterial wilt; (b) treatment of pathogen of bacterial wilt and its antagonistic bacteria.

by loading these two kinds of microbes, the loading of $R$. solanacearum did not change the content of sugars, but significantly increasedby the loading of $B$. amyloliquefacien YH22 (T-test, $p=0.03$ ), the loading of microbes did not obviously change the content of esters in three treatments (Figure 2). 
Table 1. The content of tobacco root exudates under Ralstonia solanacearum and Bacillus amyloliquefacien YH22.

\begin{tabular}{|c|c|c|c|c|}
\hline \multirow[b]{2}{*}{ Components of tobacco root exudates } & \multicolumn{4}{|c|}{ Peak area $(\%)$} \\
\hline & Molecular Formula & Control group & Pathogen of bacterial wilt & $\begin{array}{c}\text { Pathogen of } \\
\text { bacterial wilt + antagonist }\end{array}$ \\
\hline Methylamine & $\mathrm{C}_{7} \mathrm{H}_{21} \mathrm{~N}$ & $8.9354 \pm 0.29$ & $9.1213 \pm 0.26$ & $9.5606 \pm 0.25$ \\
\hline Tyramine & $\mathrm{C}_{8} \mathrm{H}_{11} \mathrm{NO}$ & $(-)$ & $2.1331 \pm 0.30$ & $1.2131 \pm 0.32$ \\
\hline Silanamine & $\mathrm{C}_{7} \mathrm{H}_{18} \mathrm{~N}_{2}$ & $(-)$ & $5.4464 \pm 0.22$ & $6.5480 \pm 0.34$ \\
\hline Bis(trimethylsilyl)carbodiimide & $\mathrm{C}_{7} \mathrm{H}_{18} \mathrm{~N}_{2}$ & $1.2014 \pm 0.35$ & $1.6521 \pm 0.29$ & $1.2091 \pm 0.25$ \\
\hline N-Methyltrifluoroacetamide & $\mathrm{C}_{3} \mathrm{H}_{4} \mathrm{~F}_{3} \mathrm{NO}$ & $0.1541 \pm 0.10$ & $0.2514 \pm 0.05$ & $0.2618 \pm 0.06$ \\
\hline Hexanol & $\mathrm{C}_{12} \mathrm{H}_{28} \mathrm{O}$ & $4.4790 \pm 0.24$ & $3.5823 \pm 0.23$ & $3.5856 \pm 0.35$ \\
\hline Ethylene glycol & $\mathrm{C}_{8} \mathrm{H}_{22} \mathrm{O}_{2}$ & $1.6186 \pm 0.22$ & $1.6312 \pm 0.32$ & $1.4583 \pm 0.23$ \\
\hline Diethylene glycol & $\mathrm{C}_{10} \mathrm{H}_{26} \mathrm{O}_{3}$ & $1.1490 \pm 0.22$ & $(-)$ & $(-)$ \\
\hline Phenylethyl alcohol & $\mathrm{C}_{8} \mathrm{H}_{10} \mathrm{O}$ & $1.1589 \pm 0.15$ & $(-)$ & $(-)$ \\
\hline Glycerol & $\mathrm{C}_{12} \mathrm{H}_{32} \mathrm{O}_{3}$ & $2.6808 \pm 0.40$ & $2.7021 \pm 0.19$ & $3.8914 \pm 0.39$ \\
\hline Thiodiglycol & $\mathrm{C}_{4} \mathrm{H}_{10} \mathrm{O}_{2} \mathrm{~S}$ & $0.1238 \pm 0.06$ & $0.2156 \pm 0.06$ & $(-)$ \\
\hline D-Pinitol & $\mathrm{C}_{7} \mathrm{H}_{14} \mathrm{O}_{6}$ & $(-)$ & $(-)$ & $0.1423 \pm 0.04$ \\
\hline D-Mannitol & $\mathrm{C}_{24} \mathrm{H}_{62} \mathrm{O}_{6}$ & $(-)$ & $1.1671 \pm 0.16$ & $2.1651 \pm 0.20$ \\
\hline Myo-Inositol & $\mathrm{C}_{6} \mathrm{H}_{12} \mathrm{O}_{6}$ & $(-)$ & $0.5698 \pm 0.14$ & $0.5412 \pm 0.11$ \\
\hline Silanol & $\mathrm{C}_{13} \mathrm{H}_{14} \mathrm{O}$ & $(-)$ & $0.1296 \pm 0.09$ & $0.2315 \pm 0.08$ \\
\hline 1-Butanol & $\mathrm{C}_{4} \mathrm{H}_{10} \mathrm{O}$ & $(-)$ & $2.5128 \pm 0.39$ & $(-)$ \\
\hline 2,3-Butanediol & $\mathrm{C}_{10} \mathrm{H}_{26} \mathrm{O}_{2}$ & $(-)$ & $0.7250 \pm 0.11$ & $0.5283 \pm 0.02$ \\
\hline 1-Monopalmitin & $\mathrm{C}_{19} \mathrm{H}_{38} \mathrm{O}_{4}$ & $(-)$ & $1.3210 \pm 0.21$ & $1.1593 \pm 0.23$ \\
\hline Isopropyl alcohol & $\mathrm{C}_{3} \mathrm{H}_{8} \mathrm{O}$ & $(-)$ & $(-)$ & $1.1452 \pm 0.17$ \\
\hline Triethylene glycol & $\mathrm{C}_{8} \mathrm{H}_{18} \mathrm{O}_{4}$ & $2.1423 \pm 0.17$ & $(-)$ & $(-)$ \\
\hline Boric acid & $\mathrm{BC}_{9} \mathrm{H}_{27} \mathrm{O}_{3}$ & $8.4826 \pm 0.24$ & $8.5092 \pm 0.23$ & $9.5069 \pm 0.33$ \\
\hline Oxalic acid & $\mathrm{C}_{2} \mathrm{H}_{2} \mathrm{O}$ & $(-)$ & $4.2156 \pm 0.43$ & $3.5623 \pm 0.35$ \\
\hline Propionic acid & $\mathrm{C}_{3} \mathrm{H}_{6} \mathrm{O}_{2}$ & $(-)$ & $3.5986 \pm 0.38$ & $(-)$ \\
\hline Palmitic acid & $\mathrm{C}_{19} \mathrm{H}_{40} \mathrm{O}_{2}$ & $(-)$ & $1.1267 \pm 0.22$ & $3.1092 \pm 0.37$ \\
\hline Hydrocinnamic acid & $\mathrm{C}_{9} \mathrm{H}_{10} \mathrm{O}_{2}$ & $(-)$ & $0.0186 \pm 0.02$ & $0.8218 \pm 0.19$ \\
\hline Stearic acid & $\mathrm{C}_{21} \mathrm{H}_{44} \mathrm{O}_{2}$ & $5.4667 \pm 0.14$ & $4.0571 \pm 0.26$ & $7.0821 \pm 0.42$ \\
\hline Isophthalic acid & $\mathrm{C}_{8} \mathrm{H}_{6} \mathrm{O}_{4}$ & $(-)$ & $2.5862 \pm 0.24$ & $(-)$ \\
\hline Terephthalic acid & $\mathrm{C}_{8} \mathrm{H}_{6} \mathrm{O}_{4}$ & $0.7792 \pm 0.25$ & $1.5281 \pm 0.25$ & $0.8589 \pm 0.18$ \\
\hline Myristic acid & $\mathrm{C}_{14} \mathrm{H}_{28} \mathrm{O}_{2}$ & $(-)$ & $0.5836 \pm 0.15$ & $(-)$ \\
\hline Itaconic acid & $\mathrm{C}_{5} \mathrm{H}_{6} \mathrm{O}_{4}$ & $(-)$ & $0.0782 \pm 0.02$ & $0.1290 \pm 0.04$ \\
\hline Benzimidate hydrochloride & $\mathrm{C}_{9} \mathrm{H}_{12} \mathrm{NC}_{\mathrm{lO}}$ & $(-)$ & $0.0576 \pm 0.02$ & $(-)$ \\
\hline Sebacic acid & $\mathrm{C}_{10} \mathrm{H}_{18} \mathrm{O}_{4}$ & $(-)$ & $0.3276 \pm 0.04$ & $(-)$ \\
\hline Lactic acid & $\mathrm{C}_{9} \mathrm{H}_{22} \mathrm{O}_{3}$ & $7.1991 \pm 0.29$ & $5.3126 \pm 0.24$ & $6.4286 \pm 0.29$ \\
\hline Benzoic acid & $\mathrm{C}_{7} \mathrm{H}_{6} \mathrm{O}_{2}$ & $1.5823 \pm 0.23$ & $3.8569 \pm 0.43$ & $1.2982 \pm 0.28$ \\
\hline Acetic acid & $\mathrm{C}_{10} \mathrm{H}_{12} \mathrm{O}_{2}$ & $2.6790 \pm 0.26$ & $3.4087 \pm 0.28$ & $4.4589 \pm 0.13$ \\
\hline
\end{tabular}




\section{Continued}

\begin{tabular}{|c|c|c|c|c|}
\hline Octanoic acid & $\mathrm{C}_{8} \mathrm{H}_{16} \mathrm{O}_{2}$ & $1.5820 \pm 0.30$ & $1.5692 \pm 0.23$ & $1.8263 \pm 0.26$ \\
\hline 3,4-Dimethoxybenzoic acid & $\mathrm{C}_{9} \mathrm{H}_{10} \mathrm{O}_{4}$ & $(-)$ & $(-)$ & $0.0350 \pm 0.01$ \\
\hline Dehydroabietic acid & $\mathrm{C}_{20} \mathrm{H}_{28} \mathrm{O}_{2}$ & $(-)$ & $(-)$ & $1.0464 \pm 0.13$ \\
\hline Caffeic acid & $\mathrm{C}_{9} \mathrm{H}_{8} \mathrm{O}_{4}$ & $(-)$ & $(-)$ & $0.0805 \pm 0.04$ \\
\hline 3-Hydroxybutyricacid & $\mathrm{C}_{4} \mathrm{H}_{8} \mathrm{O}_{3}$ & $(-)$ & $(-)$ & $0.0428 \pm 0.01$ \\
\hline Sucrose & $\mathrm{C}_{12} \mathrm{H}_{22} \mathrm{O}_{11}$ & $(-)$ & $(-)$ & $0.0025 \pm 0.0009$ \\
\hline D-Trehalose & $\mathrm{C}_{12} \mathrm{H}_{22} \mathrm{O}_{11}$ & $(-)$ & $1.3090 \pm 0.17$ & $0.1690 \pm 0.13$ \\
\hline Galactopyranose & $\mathrm{C}_{6} \mathrm{H}_{12} \mathrm{O}_{6}$ & $(-)$ & $(-)$ & $0.0158 \pm 0.006$ \\
\hline D-(+)-Mannose & $\mathrm{C}_{6} \mathrm{H}_{12} \mathrm{O}_{6}$ & $(-)$ & $0.0431 \pm 0.01$ & $0.0543 \pm 0.01$ \\
\hline Phenethyl acetate & $\mathrm{C}_{10} \mathrm{H}_{12} \mathrm{O}_{2}$ & $5.4376 \pm 0.26$ & $5.0627 \pm 0.25$ & $5.0289 \pm 0.21$ \\
\hline Valtrate & $\mathrm{C}_{22} \mathrm{H}_{30} \mathrm{O}_{8}$ & $(-)$ & $(-)$ & $0.5030 \pm 0.07$ \\
\hline Glycerol monostearate & $\mathrm{C}_{21} \mathrm{H}_{42} \mathrm{O}_{4}$ & $(-)$ & $(-)$ & $0.3082 \pm 0.03$ \\
\hline Dodecanoic acid & $\mathrm{C}_{24} \mathrm{H}_{48} \mathrm{O}_{2}$ & $(-)$ & $(-)$ & $0.0150 \pm 0.03$ \\
\hline Propyl heptanoate & $\mathrm{C}_{10} \mathrm{H}_{20} \mathrm{O}_{2}$ & $(-)$ & $0.0591 \pm 0.008$ & $0.0523 \pm 0.007$ \\
\hline Cyclopropane & $\mathrm{C}_{3} \mathrm{H}_{6}$ & $(-)$ & $0.0112 \pm 0.009$ & $(-)$ \\
\hline Urea & $\mathrm{CH}_{4} \mathrm{~N}_{2} \mathrm{O}$ & $0.0039 \pm 0.001$ & $0.0036 \pm 0.0009$ & $0.0153 \pm 0.001$ \\
\hline
\end{tabular}

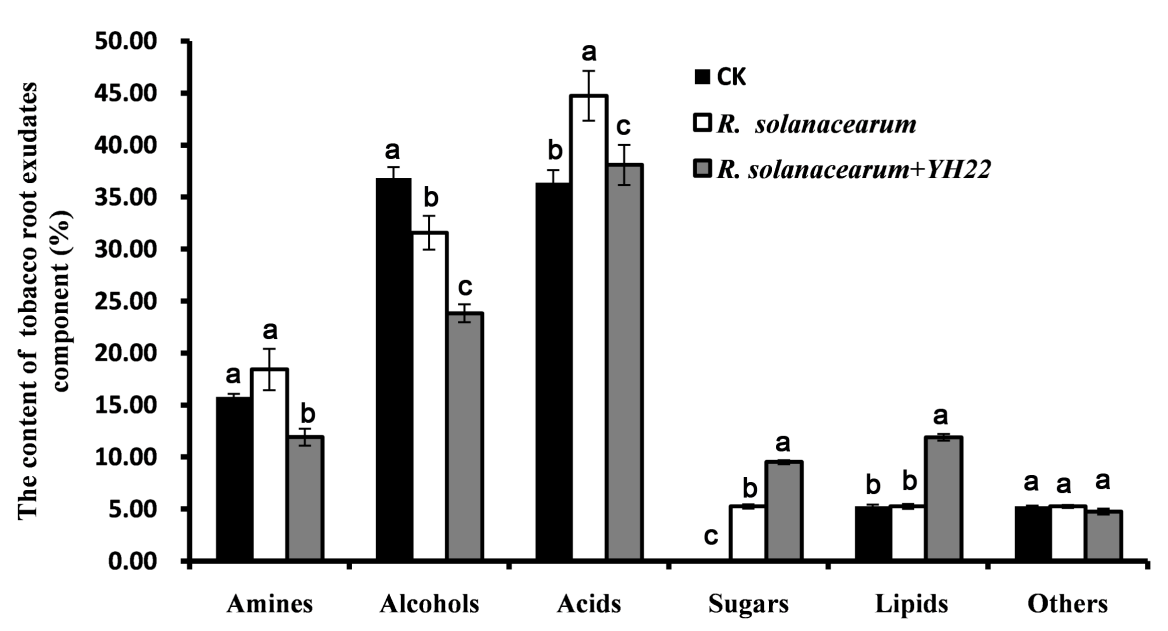

Figure 2. Changes of tobacco root exudates after treatment of bacterial wilt pathogen and its antagonist ((a), (b), (c) represent significant difference).

2) Analysis of tobacco root exudates after treating by pathogen of black shank and its antagonist.

The composition of the root exudates of Yunyan 87 was analyzed by GC-MS after irrigating pathogen of black shank and its antagonist (Figure 3). Under the condition of the pathogen of black shank, the contents of amines, alcohols, acids, lipids, sugars and esters were $14.28 \%, 28.57 \%, 48.57 \%, 2.85 \%, 2.85 \%$ and $2.85 \%$ respectively. By the treatment of antagonistic bacteria of black shank, the contents of amines, alcohols, acids, lipids, sugars and esters were 11.36\%, 22.72\%, 


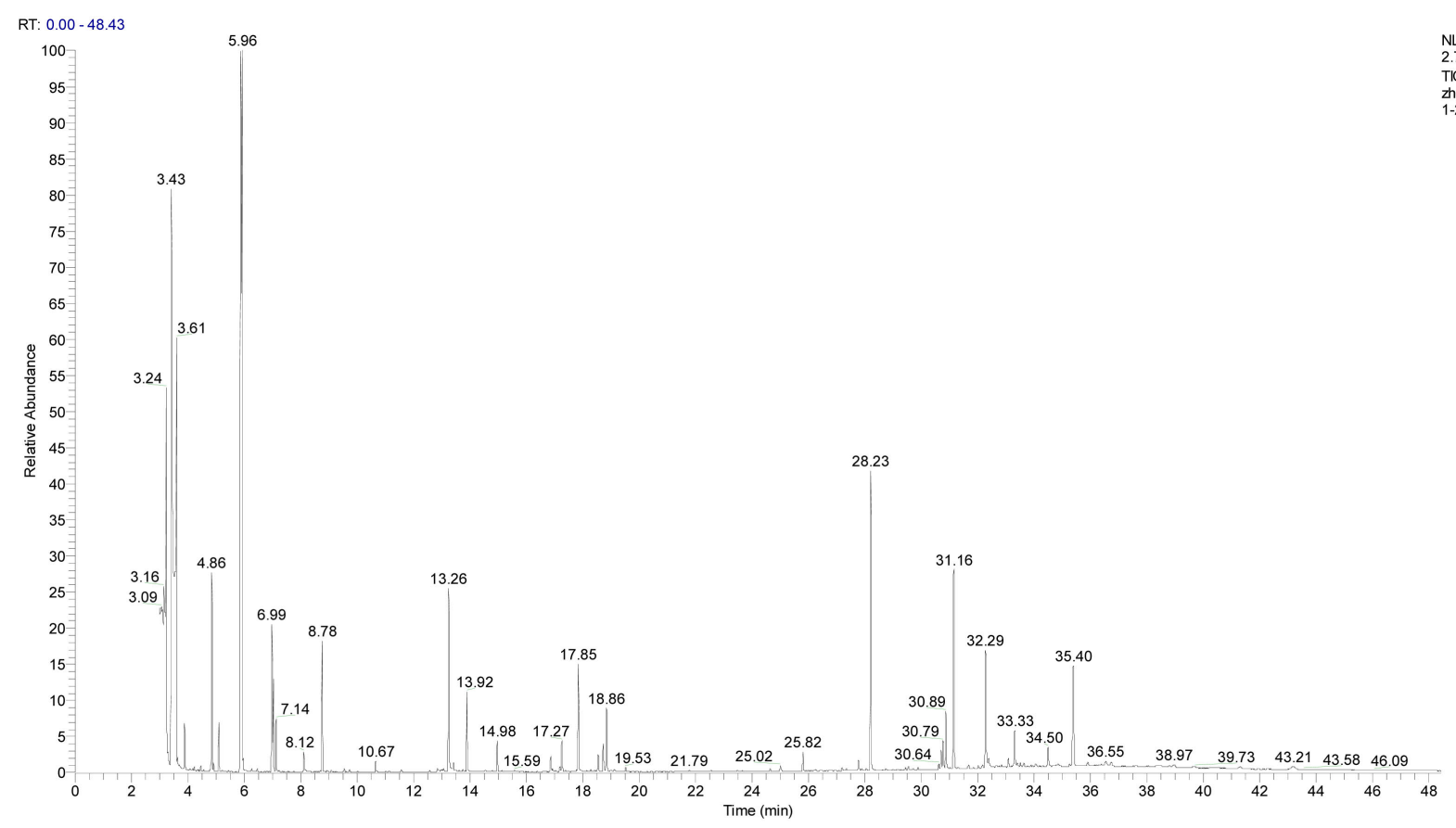

(a)

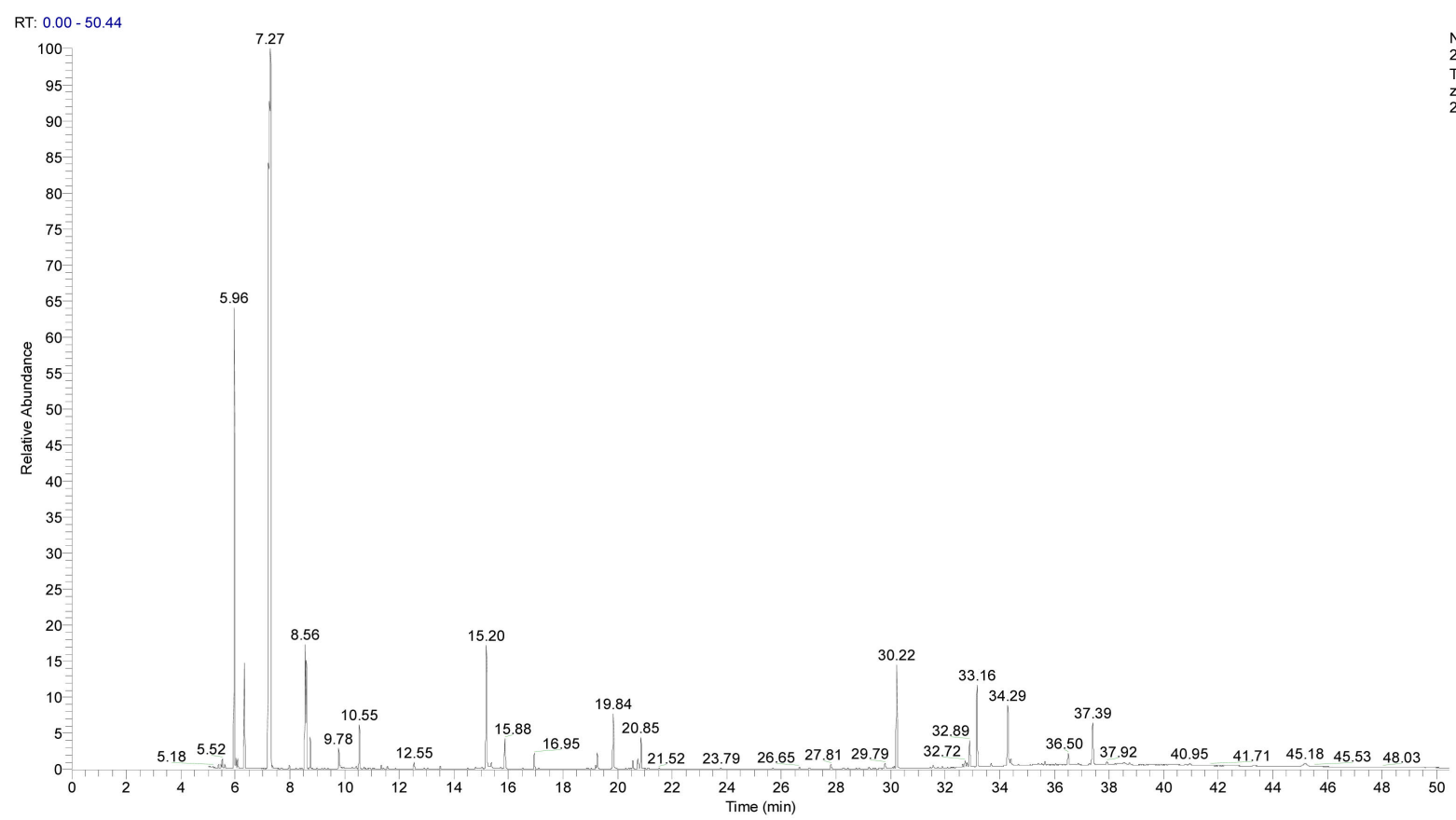

(b)

Figure 3. Total ion current of GC-MS with (a) treatment of pathogen of black shank; (b) treatment of pathogen of black shank and its antagonistic bacteria.

$36.36 \%, 6.81 \%, 11.36 \%$ and $11.36 \%$ separately. There were more than 20 types of root exudates of Yunyan 87 compared with the control group, mainly including 2 kinds of amines, 5 kinds of alcohols, 11 kinds of acids, 1 kind of sugars, 1 kind of esters respectively (Table 2). There are the same components of 8 types with contents decreased and 4 types with contents increased. It was noteworthy that 
Table 2. The content of tobacco root exudates under Phytophthora parasitica and Bacillus amyloliquefacien TU1.

\begin{tabular}{|c|c|c|c|c|}
\hline \multirow[b]{2}{*}{ Components of tobacco root exudates } & \multicolumn{4}{|c|}{ Peak area $(\%)$} \\
\hline & Formula & Control group & Pathogen of black shank & $\begin{array}{l}\text { Pathogen of black } \\
\text { shank + antagonist }\end{array}$ \\
\hline Methylamine & $\mathrm{C}_{7} \mathrm{H}_{21} \mathrm{~N}$ & $8.9354 \pm 0.58$ & $8.6354 \pm 0.43$ & $8.1213 \pm 0.46$ \\
\hline Tyramine & $\mathrm{C}_{8} \mathrm{H}_{11} \mathrm{NO}$ & $(-)$ & $2.0031 \pm 0.36$ & $1.6131 \pm 0.30$ \\
\hline Silanamine & $\mathrm{C}_{7} \mathrm{H}_{18} \mathrm{~N}_{2}$ & $(-)$ & $4.3898 \pm 0.27$ & $5.3218 \pm 0.33$ \\
\hline Bis(trimethylsilyl)carbodiimide & $\mathrm{C}_{7} \mathrm{H}_{18} \mathrm{~N}_{2}$ & $1.2014 \pm 0.22$ & $1.1304 \pm 0.34$ & $1.9807 \pm 0.42$ \\
\hline N-Methyltrifluoroacetamide & $\mathrm{C}_{3} \mathrm{H}_{4} \mathrm{~F}_{3} \mathrm{NO}$ & $0.1541 \pm 0.16$ & $1.3543 \pm 0.31$ & $0.3318 \pm 0.19$ \\
\hline Hexanol & $\mathrm{C}_{12} \mathrm{H}_{28} \mathrm{O}$ & $4.4790 \pm 0.42$ & $3.5823 \pm 0.52$ & $4.5998 \pm 0.35$ \\
\hline Ethylene glycol & $\mathrm{C}_{8} \mathrm{H}_{22} \mathrm{O}_{2}$ & $1.6186 \pm 0.31$ & $1.1563 \pm 0.25$ & $1.2380 \pm 0.32$ \\
\hline Diethylene glycol & $\mathrm{C}_{10} \mathrm{H}_{26} \mathrm{O}_{3}$ & $1.1490 \pm 0.43$ & $(-)$ & $(-)$ \\
\hline Phenylethyl alcohol & $\mathrm{C}_{8} \mathrm{H}_{10} \mathrm{O}$ & $1.1589 \pm 0.44$ & $(-)$ & $(-)$ \\
\hline Glycerol & $\mathrm{C}_{12} \mathrm{H}_{32} \mathrm{O}_{3}$ & $2.6808 \pm 0.68$ & $2.0565 \pm 0.41$ & $1.9967 \pm 0.11$ \\
\hline Thiodiglycol & $\mathrm{C}_{4} \mathrm{H}_{10} \mathrm{O}_{2} \mathrm{~S}$ & $0.1238 \pm 0.08$ & $0.1615 \pm 0.11$ & $(-)$ \\
\hline D-Pinitol & $\mathrm{C}_{7} \mathrm{H}_{14} \mathrm{O}_{6}$ & $(-)$ & $(-)$ & $0.1120 \pm 0.04$ \\
\hline D-Mannitol & $\mathrm{C}_{24} \mathrm{H}_{62} \mathrm{O}_{6}$ & $(-)$ & $1.6160 \pm 0.28$ & $2.0615 \pm 0.33$ \\
\hline Myo-Inositol & $\mathrm{C}_{6} \mathrm{H}_{12} \mathrm{O}_{6}$ & $(-)$ & $0.2298 \pm 0.17$ & $0.0982 \pm 0.06$ \\
\hline Silanol & $\mathrm{C}_{13} \mathrm{H}_{14} \mathrm{O}$ & $(-)$ & $0.2629 \pm 0.14$ & $0.3234 \pm 0.08$ \\
\hline 1-Butanol & $\mathrm{C}_{4} \mathrm{H}_{10} \mathrm{O}$ & $(-)$ & $2.5342 \pm 0.38$ & $(-)$ \\
\hline 2,3-Butanediol & $\mathrm{C}_{10} \mathrm{H}_{26} \mathrm{O}_{2}$ & $(-)$ & $0.0830 \pm 0.05$ & $0.5429 \pm 0.19$ \\
\hline 1-Monopalmitin & $\mathrm{C}_{19} \mathrm{H}_{38} \mathrm{O}_{4}$ & $(-)$ & $1.0321 \pm 0.29$ & $1.1421 \pm 0.29$ \\
\hline Isopropyl alcohol & $\mathrm{C}_{3} \mathrm{H}_{8} \mathrm{O}$ & $(-)$ & $(-)$ & $1.0852 \pm 0.27$ \\
\hline Triethylene glycol & $\mathrm{C}_{8} \mathrm{H}_{18} \mathrm{O}_{4}$ & $2.1423 \pm 0.34$ & $(-)$ & $(-)$ \\
\hline Boric acid & $\mathrm{BC}_{9} \mathrm{H}_{27} \mathrm{O}_{3}$ & $8.4826 \pm 0.31$ & $8.5014 \pm 0.37$ & $9.5421 \pm 0.25$ \\
\hline Oxalic acid & $\mathrm{C}_{2} \mathrm{H}_{2} \mathrm{O}$ & $(-)$ & $4.6245 \pm 0.53$ & $3.5460 \pm 0.54$ \\
\hline Propionic acid & $\mathrm{C}_{3} \mathrm{H}_{6} \mathrm{O}_{2}$ & $(-)$ & $1.5436 \pm 0.28$ & $(-)$ \\
\hline Oleic acid & $\mathrm{C}_{21} \mathrm{H}_{42} \mathrm{O}_{2}$ & $(-)$ & $0.0743 \pm 0.04$ & $0.0628 \pm 0.05$ \\
\hline Palmitic acid & $\mathrm{C}_{19} \mathrm{H}_{40} \mathrm{O}_{2}$ & $(-)$ & $1.2187 \pm 0.23$ & $3.0752 \pm 0.29$ \\
\hline Hydrocinnamic acid & $\mathrm{C}_{9} \mathrm{H}_{10} \mathrm{O}_{2}$ & $(-)$ & $0.0567 \pm 0.04$ & $0.1822 \pm 0.14$ \\
\hline Stearic acid & $\mathrm{C}_{21} \mathrm{H}_{44} \mathrm{O}_{2}$ & $5.4667 \pm 0.36$ & $3.9579 \pm 0.29$ & $5.0162 \pm 0.37$ \\
\hline Isophthalic acid & $\mathrm{C}_{8} \mathrm{H}_{6} \mathrm{O}_{4}$ & $(-)$ & $2.5536 \pm 0.30$ & $(-)$ \\
\hline Terephthalic acid & $\mathrm{C}_{8} \mathrm{H}_{6} \mathrm{O}_{4}$ & $0.7792 \pm 0.36$ & $1.2501 \pm 0.27$ & $0.8705 \pm 0.35$ \\
\hline Myristic acid & $\mathrm{C}_{14} \mathrm{H}_{28} \mathrm{O}_{2}$ & $(-)$ & $1.5432 \pm 0.29$ & $(-)$ \\
\hline ITACONIC ACID & $\mathrm{C}_{5} \mathrm{H}_{6} \mathrm{O}_{4}$ & $(-)$ & $0.1482 \pm 0.10$ & $1.1209 \pm 0.30$ \\
\hline Benzimidate hydrochloride & $\mathrm{C}_{9} \mathrm{H}_{12} \mathrm{NC}_{1 \mathrm{l}}$ & $(-)$ & $0.0942 \pm 0.05$ & $(-)$ \\
\hline Sebacic Acid & $\mathrm{C}_{10} \mathrm{H}_{18} \mathrm{O}_{4}$ & $(-)$ & $0.8764 \pm 0.24$ & $(-)$ \\
\hline Lactic Acid & $\mathrm{C}_{9} \mathrm{H}_{22} \mathrm{O}_{3}$ & $7.1991 \pm 0.42$ & $3.2412 \pm 0.26$ & $2.4098 \pm 0.26$ \\
\hline Benzoic acid & $\mathrm{C}_{7} \mathrm{H}_{6} \mathrm{O}_{2}$ & $1.5823 \pm 0.34$ & $2.2651 \pm 0.34$ & $1.0092 \pm 0.15$ \\
\hline
\end{tabular}




\begin{tabular}{|c|c|c|c|c|}
\hline Acetic acid & $\mathrm{C}_{10} \mathrm{H}_{12} \mathrm{O}_{2}$ & $2.6790 \pm 0.28$ & $3.8057 \pm 0.35$ & $4.4490 \pm 0.27$ \\
\hline Octanoic acid & $\mathrm{C}_{8} \mathrm{H}_{16} \mathrm{O}_{2}$ & $1.5820 \pm 0.29$ & $1.9920 \pm 0.74$ & $1.8836 \pm 0.34$ \\
\hline 3,4-Dimethoxybenzoic acid & $\mathrm{C}_{9} \mathrm{H}_{10} \mathrm{O}_{4}$ & $(-)$ & $(-)$ & $0.6530 \pm 0.31$ \\
\hline Dehydroabietic acid & $\mathrm{C}_{20} \mathrm{H}_{28} \mathrm{O}_{2}$ & $(-)$ & $(-)$ & $1.0472 \pm 0.09$ \\
\hline Caffeic acid & $\mathrm{C}_{9} \mathrm{H}_{8} \mathrm{O}_{4}$ & $(-)$ & $(-)$ & $0.1054 \pm 0.03$ \\
\hline 3-Hydroxybutyricacid & $\mathrm{C}_{4} \mathrm{H}_{8} \mathrm{O}_{3}$ & $(-)$ & $(-)$ & $0.0855 \pm 0.07$ \\
\hline Sucrose & $\mathrm{C}_{12} \mathrm{H}_{22} \mathrm{O}_{11}$ & $(-)$ & $(-)$ & $0.0355 \pm 0.003$ \\
\hline D-Trehalose & $\mathrm{C}_{12} \mathrm{H}_{22} \mathrm{O}_{11}$ & $(-)$ & $(-)$ & $(-)$ \\
\hline Galactopyranose & $\mathrm{C}_{6} \mathrm{H}_{12} \mathrm{O}_{6}$ & $(-)$ & $(-)$ & $0.0238 \pm 0.002$ \\
\hline D-(+)-Mannose & $\mathrm{C}_{6} \mathrm{H}_{12} \mathrm{O}_{6}$ & $(-)$ & $0.1233 \pm 0.08$ & $0.0945 \pm 0.30$ \\
\hline Phenethyl acetate & $\mathrm{C}_{10} \mathrm{H}_{12} \mathrm{O}_{2}$ & $5.4376 \pm 0.68$ & $5.3637 \pm 0.32$ & $5.3490 \pm 0.29$ \\
\hline Valtrate & $\mathrm{C}_{22} \mathrm{H}_{30} \mathrm{O}_{8}$ & $(-)$ & $(-)$ & $0.5789 \pm 0.26$ \\
\hline Glycerol monostearate & $\mathrm{C}_{21} \mathrm{H}_{42} \mathrm{O}_{4}$ & $(-)$ & $(-)$ & $0.8582 \pm 0.25$ \\
\hline Dodecanoic acid & $\mathrm{C}_{24} \mathrm{H}_{48} \mathrm{O}_{2}$ & $(-)$ & $(-)$ & $0.0952 \pm 0.03$ \\
\hline Propyl heptanoate & $\mathrm{C}_{10} \mathrm{H}_{20} \mathrm{O}_{2}$ & $(-)$ & $0.0589 \pm 0.04$ & $0.1509 \pm 0.03$ \\
\hline Urea & $\mathrm{CH}_{4} \mathrm{~N}_{2} \mathrm{O}$ & $0.0039 \pm 0.001$ & $0.0136 \pm 0.002$ & $0.0134 \pm 0.003$ \\
\hline
\end{tabular}

the most remarkable increase was terephthalic acid (60.43\%) and benzoic acid $(43.15 \%)$. After the addition of antagonistic bacteria of black shank, there were 24 kinds of components increased among of which 2 were newly appeared and 13 of them decreased among of which 7 were completely disappeared, namely thioethylene glycol, 1-butanol, propionic acid, isophthalic acid, myristic acid, ethyl benzoate and azelaic acid (Figure 4).

According to the analysis, we found that the content of propionic acid, isophthalic acid, myristic acid, ethyl benzoate and azelaic acid showed the greatest volatility, the content in the control group was 0 . The treatment group of pathogen of black shank reached the highest value, while the content decreased to 0 after the addition of antagonistic bacteria, followed by terephthalic acid and benzoic acid, which increased from 0.78 to 1.53 and 2.27 respectively, and decreased to 0.86 and 1.01 after adding antagonistic bacteria. Totally, amines were significantly decreased by loading B. amyloliquefacien TU1 (T-test, $p=0.048$ ), alcohols content were continuously and significantly decreased by loading $P$. parasitica var. nicotianae and its antagonistic bacteria Bacillus amyloliquefacien TU1 (T-test, $p=0.03$ ), the content of acids and lipids were alwalys significantly increased by loading these two kinds of microbes, the loading of $P$. parasitica var. nicotianae did not change the content of sugars, but significantly increasedby the loading of $B$. amyloliquefacien TU1 (T-test, $t=0.02$ ), the loading of microbes did not obviously change the content of esters in three treatments (Figure 4). 


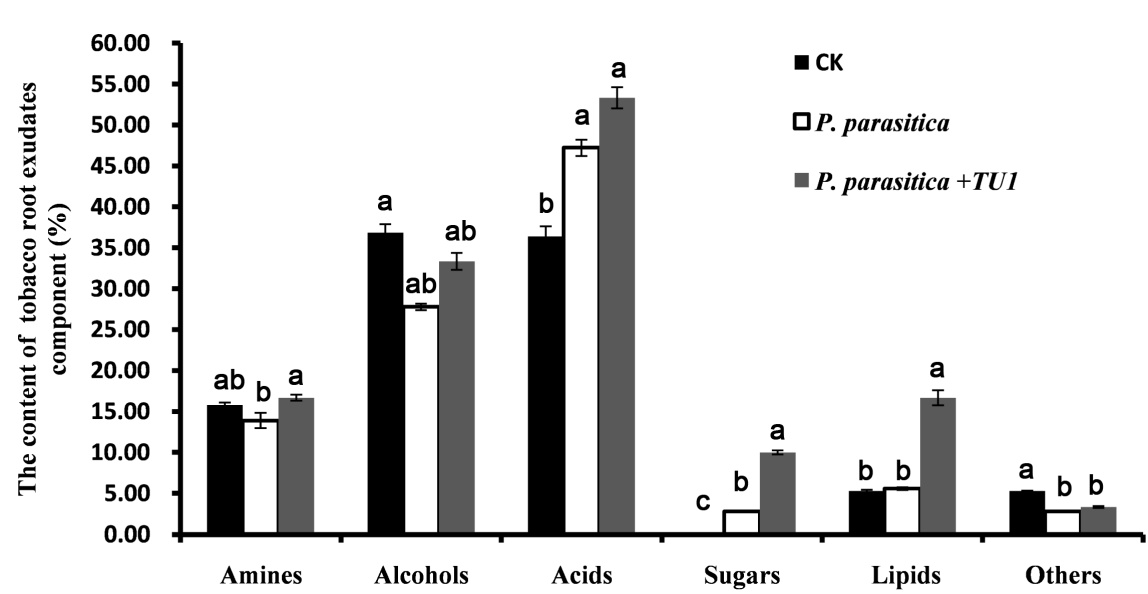

Figure 4. Changes of tobacco root exudates after treatment of black shank pathogen and its antagonist ((a), (b), (c) represent significant difference).

Plant-root exudates-rhizosphere microbes form a cyclical interaction [23], and rhizosphere microorganisms convert organic nutrients of root exudates into inorganic nutrients to facilitate plant utilization, thus affecting comprehensive indicators of plants [24] [25] [26] [27]. By analyzing the changes of root exudates of tobacco under the effect of different microbes, it was found that when the pathogenic microorganisms in the rhizosphere microorganisms increased, the root exudates of tobacco augmented evidently. When the abundance of pathogens of bacterial wilt in the rhizosphere increased, the organic acids, amines and lipids increased by $32.78 \%, 13.35 \%$ and $15.76 \%$ respectively among which organic acids displayed the greatest rise, increased by $10.11 \%$; followed by amine, increased by $6.09 \%$. When the pathogen of bacterial wilt was inhibited by the active substances of its antagonistic bacteria, the root exudates changed significantly, and there were newly 19 kinds of elements added, but 1-butanol, propionic acid, isophthalic acid, myristic acid, phenethyl imine acid, sebacic acid and cyclopropene disappeared. Although the mechanism of effect of propionic acid, myristic acid, ethyl benzoate and sebacic acid was unclear, studies have confirmed that terephthalic acid, isophthalic acid and benzoic acid are allelopathic substances to many crops. They are crucial substance that cause continuous cropping obstacles [28] [29] [30] [31] [32]. When the abundance of pathogen of bacterial wilt and its antagonist changed in the rhizosphere, the changes of root exudates were similar to the treatment of bacterial wilt and its antagonist.

There are some differences in the number of tobacco root exudates under different treatments. The proportion of the amount is shown in Figure 2 and Figure 4, but the types of secretions are mainly amines, alcohols, organic acids and sugars. In the control group of tobacco root exudates, there was no carbohydrate, but it was detected in the treatment group of pathogen of bacterial wilt. After adding antagonistic bacteria, the types of carbohydrates increased and the change of organic acids was the most obvious: the control group was $36.84 \%$, and the treatment group of bacterial wilt pathogen was $44.74 \%$. Under the in- 
fluence of pathogenic bacteria and antagonist, the types of organic acid increased to $51.61 \%$, with a rise of $21.44 \%$ and $15.35 \%$ respectively. The change rule of root exudates of black shank pathogen and its antagonist was similar to that of bacterial wilt pathogen and its antagonist. However, the types of organic acid increased to $47.22 \%$ and $53.33 \%$, with a rose of $28.18 \%$ and $12.93 \%$. Data has shown that through the treatment of bacterial wilt, black shank pathogen and their antagonistic bacteria, the content of propionic acid, isophthalic acid, myristic acid, ethyl benzoate and azelaic acid fluctuated abundantly, while the control group was 0 . The treatment group of bacterial wilt pathogen reached the highest its peak, and the content decreased to 0 after the addition of antagonistic bacteria, followed by terephthalic acid and benzoic acid, which increased from 0.78 to 1.53 and 2.27 respectively, yet decreased to 0.86 and 1.01 separately after adding antagonistic bacteria. The results showed that propionic acid, isophthalic acid, myristic acid, ethyl benzoate and azelaic acid were direct inducers of bacterial wilt and black shank pathogens, and their antagonistic bacteria could alleviate harmful bacteria that induced tobacco to produce allelochemicals (terephthalic acid, isophthalic acid and benzoic acid). The dramatical increase of the abundance of pathogen in soil was a vital factor which led to continuous cropping obstacles. It was speculated that the possible mechanism was due to the increase of the abundance of pathogen [33], which induced crops to produce allelopathic substances, thus allelochemicals provide nutrients for the colonization of harmful bacteria. It formed a vicious circle that eventually resulted in continuous cropping obstacles. Although this conjecture needs further confirmation, the study provides new clues for exploring tobacco continuous cropping disorders and soil-borne diseases.

\section{Acknowledgements}

We wish to thank State Key Laboratory of Biocatalysis and Enzyme Engineering for providing the Laboratory space and equipments required for the research work. This work also has been supported by China National Tobacco Corporation, Grant (110201502018, 110201502014 and 027Y2014-013).

\section{Conflicts of Interest}

The authors declare no conflicts of interest regarding the publication of this paper.

\section{References}

[1] Berendsen, R.L., Pieterse, C.M.J., Bakker, P.A.H.M., et al. (2012) The Rhizosphere Microbiome and Plant Health. Trends in Plant Science, 17, 478-486.

https://doi.org/10.1016/j.tplants.2012.04.001

[2] Egamberdieva, D., Kamilova, F., Validov, S., et al. (2008) High Incidence of Plant Growth-Stimulating Bacteria Associated with the Rhizosphere of Wheat Grown on Salinated Soil in Uzbekistan. Environmental Microbiology, 10, 1-9.

[3] Mendes, R., Kruijt, M., de Bruijn, I., et al. (2011) Deciphering the Rhizosphere Mi- 
crobiome for Disease-Suppressive Bacteria. Science, 332, 1097-1100. https://doi.org/10.1126/science.1203980

[4] Pradeep, R.M. (2015) The Promise of the Plant's Second Genome. Journal of Investigative Genomics, 2, 83.

[5] Zamioudis, C. and Pieterse, C.M. (2012) Modulation of Host Immunity by Beneficial Microbes. Molecular Plant-Microbe Interactions, 25, 139-150. https://doi.org/10.1094/MPMI-06-11-0179

[6] Zhang, Y., Du, B.H., Jin, Z.G., et al. (2011) Analysis of Bacterial Communities in Rhizosphere Soil of Healthy and Diseased Cotton (Gossypium sp.) at Different Plant Growth Stages. Plant Soil, 339, 447-455. https://doi.org/10.1007/s11104-010-0600-2

[7] Lanoue, A., Burlat, V., Henkes, G.J., et al. (2010) De Novo Biosynthesis of Defense Root Exudates in Response to Fusarium Attack in Barley. New Phytologist, 185, 577-588. https://doi.org/10.1111/j.1469-8137.2009.03066.x

[8] Yang, J.W., Yi, H.S., Kim, H., et al. (2011) Whitefly Infestation of Pepper Plants Elicits Defence Responses against Bacterial Pathogens in Leaves and Roots and Changes the Below-Ground Microflora. Journal of Ecology, 99, 46-56.

https://doi.org/10.1111/j.1365-2745.2010.01756.x

[9] Lee, B., Lee, S. and Ryu, C.M. (2012) Foliar Aphid Feeding Recruits Rhizosphere Bacteria and Primes Plant Immunity against Pathogenic and Non-Pathogenic Bacteria in Pepper. Annals of Botany, 110, 281-290.

https://doi.org/10.1093/aob/mcs055

[10] Zhang, L., Xu, H.M. and Zhu, B.L. (2016) Soil Microbiome with the Occurrence and Development of Replant Disease-A Review. Acta Microbiologica Sinica, 56, 1234-1241.

[11] Sasse, J., Martinoia, E. and Northen, T. (2018) Feed Your Friends: Do Plant Exudates Shape the Root Microbiome? Trends in Plant Science, 23, 25-41. https://doi.org/10.1016/j.tplants.2017.09.003

[12] Bais, H.P., Weir, T.L., Perry, L.G., et al. (2006) The Role of Root Exudates in Rhizosphere Interactions with Plants and Other Organisms. Annual Review of Plant Biology, 57, 233-266. https://doi.org/10.1146/annurev.arplant.57.032905.105159

[13] Weisskopf, L., Le, B.R., Kohler, F., et al. (2008) Spatio-Temporal Dynamics of Bacterial Communities Associated with Two Plant Species Differing in Organic Acid Secretion: A One-Year Microcosm Study on Lupin and Wheat. Soil Biology and Biochemistry, 40, 1772-1780. https://doi.org/10.1016/j.soilbio.2008.02.018

[14] He, H., Wang, Z.W., Hu, D., et al. (2011) Progress on Interactions between Root Exudates and Rhizosphere Microorganisms. Journal of Hebei Agricultural Sciences, $15,69-73$.

[15] Kawasaki, A., Suzanne, D., Peter, R., et al. (2016) Microbiome and Exudates of the Root and Rhizosphere of Brachypodium distachyon, a Modelfor Wheat. PLoS ONE, 11, e0164533. https://doi.org/10.1371/journal.pone.0164533

[16] Raaijmakers, J.M., Paulitz, T.C., Steinberg, C., et al. (2009) The Rhizosphere: A Playground and Battlefield for Soilborne Pathogens and Beneficial Microorganisms. Plant Soil, 321, 341-361. https://doi.org/10.1007/s11104-008-9568-6

[17] Hoitink, H. and Boehm, M. (1999) Biocontrol within the Context of Soil Microbial Communities: A Substrate-Dependent Phenomenon. Annual Review of Phytopathology, 37, 427-446. https://doi.org/10.1146/annurev.phyto.37.1.427

[18] Garbeva, P., Veen, J.A., Elsas, J.D., et al. (2004) Microbial Diversity in Soil: Selection 
of Microbial Populations by Plant and Soil Type and Implications for Disease Suppressiveness. Annual Review of Phytopathology, 42, 243-270. https://doi.org/10.1146/annurev.phyto.42.012604.135455

[19] Weller, D.M., Raaijmakers, J.M., Gardener, B.B., et al. (2002) Microbial Populations Responsible for Specific Soil Suppressiveness to Plant Pathogens. Annual Review of Phytopathology, 40, 309-348. https://doi.org/10.1146/annurev.phyto.40.030402.110010

[20] Gagne, B.F., Mayer, B.F., Charron, J.B., et al. (2015) Accelerated Growth Rate and Increased Drought Stress Resilience of the Model Grass Brachypodium Distachyon Colonized by Bacillus subtilis B26. PLOS ONE, 10, 11-16.

[21] Amaral, F.P., Pankievicz, V.C., Arisi, A.C., et al. (2016) Differential Growth Responses of Brachypodium distachyon Genotypes to Inoculation with Plant Growth Promoting Rhizobacteria. Plant Molecular Biology, 90, 689-697. https://doi.org/10.1007/s11103-016-0449-8

[22] Yu, H., Shen, G. and Gao, X. (2013) Determination of Tobacco Root Exudates by GC-MS. Acta Tabacaria Sinica, 19, 64-71.

[23] Shi, S.J., Richardson, A.E., O’Callaghan, M., et al. (2011) Elects of Selected Root Exudate Components on Soil Bacterial Communities. FEMS Microbiology Ecology, 77, 600-610. https://doi.org/10.1111/j.1574-6941.2011.01150.x

[24] Zhang, A., Sun, K., Da, W., et al. (2008) Study on the Microorganism of Two Habitats of Hippophae rhamnoides Rhizosphere Soil. Journal of Northwest Normal University (Natural Science), 44, 69-73.

[25] Lu, Y. and Zhang, S. (2006) Progress on Rhizosphere Microorganisms. Soil, 38, 113-121.

[26] Chen, L., Li, P., Si, L., et al. (2002) Ecological Research of Root Exudates. Journal of Ecology, 21, 57-62.

[27] Graham, R.D. (1988) Genotypic Differences in Tolerance to Manganese Deficiency. In: Zinic in Soil and Plants, Kluwer Academic Publishers, Dordrecht, 78-85.

[28] Liu, H., Song, Q. and Li, F. (2002) The Roles of Root Exudation on Rhizosphere Nutrient and Rhizosphere Microorganisms. Acta Botanica Boreali-Occidentalia Sinica, 22, 693-702.

[29] Wang, F. (2017) Effect of 1,2-Benzenedicarboxylic Acid on Root Growth of Adzuki Bean. Qinghai Agriculture and Forestry Science and Technology, 4, 12-16.

[30] Liu, Y., Li, X., Cai, L., et al. (2016) Identification of Phenolic Acids in Tobacco Root Exudates and Their Role in the Growth of Rhizosphere Microorganisms. Journal of Plant Nutrition and Fertilizer, 22, 418-428.

[31] He, Z., Wang, R., Wang, X., et al. (2014) Preliminary Study of Screening of Peanut Autotoxicity Degrading Microorganism and the Degradation Effect. Chinese Agricultural Science Bulletin, 30, 224-227.

[32] Geng, G., Zhang, S. and Cheng, Z. (2008) Allelopathy of 1,2-Benzenedicarboxylic Acid and Its Mechanism. Journal of Hunan Agricultural University (Natural Sciences), 34, 656-659.

[33] Gu, Y., Qiu, Q., Wang, Z., et al. (2012) Effects of Soybean Continuous Cropping on Microbial and Soil Enzymes in Soybean Rhizosphere. Scientia Agricultura Sinica, 45, 3955-3964. 